\title{
Retronasal Odor Representations in the Dorsal Olfactory Bulb of Rats
}

\author{
Shree Hari Gautam and Justus V. Verhagen \\ The John B. Pierce Laboratory and Department of Neurobiology, Yale University School of Medicine, New Haven, Connecticut 06519
}

\begin{abstract}
Animals perceive their olfactory environment not only from odors originating in the external world (orthonasal route) but also from odors released in the oral cavity while eating food (retronasal route). Retronasal olfaction is crucial for the perception of food flavor in humans. However, little is known about the retronasal stimulus coding in the brain. The most basic questions are if and how route affects the odor representations at the level of the olfactory bulb $(\mathrm{OB})$, where odor quality codes originate. We used optical calcium imaging of presynaptic dorsal $\mathrm{OB}$ responses to odorants in anesthetized rats to ask whether the rat $\mathrm{OB}$ could be activated retronasally, and how these responses compare to orthonasal responses under similar conditions. We further investigated the effects of specific odorant properties on orthonasal versus retronasal response patterns. We found that at a physiologically relevant flow rate, retronasal odorants can effectively reach the olfactory receptor neurons, eliciting glomerular response patterns that grossly overlap with those of orthonasal responses, but differ from the orthonasal patterns in the response amplitude and temporal dynamics. Interestingly, such differences correlated well with specific odorant properties. Less volatile odorants yielded relatively smaller responses retronasally, but volatility did not affect relative temporal profiles. More polar odorants responded with relatively longer onset latency and time to peak retronasally, but polarity did not affect relative response magnitudes. These data provide insight into the early stages of retronasal stimulus coding and establish relationships between orthonasal and retronasal odor representations in the rat $\mathrm{OB}$.
\end{abstract}

\section{Introduction}

In mammals, odorants can reach the olfactory receptor neurons (ORNs) by two routes: orthonasally, when volatiles enter the nasal cavity during inhalation/sniffing; and retronasally, when food volatiles released in the mouth pass into the nasal cavity during exhalation/eating. Rozin (1982) considered these routes as two distinct modes of olfaction and hypothesized that the perception of odorants depends on odor route. Several psychophysical (Pierce and Halpern, 1996; Heilmann and Hummel, 2004; Sun and Halpern, 2005) and human brain imaging (Small et al., 2005) studies support this hypothesis, and suggest that orthonasal and retronasal delivery of the same odorant evokes distinct perceptions and patterns of neural response in the cortical areas of the brain. However, mechanisms underlying these differences remain unknown. The most fundamental question is if and how orthonasal and retronasal odor representations differ from each other at the level of the olfactory bulb (OB), the first synaptic relay in the olfactory system, where odor quality codes originate

Received March 22, 2012; revised April 12, 2012; accepted April 22, 2012.

Author contributions:S.H.G. and J.V.V. designed research;S.H.G. and J.V.V. performed research; S.H.G. and J.V.V. contributed unpublished reagents/analytic tools; S.H.G. and J.V.V. analyzed data; S.H.G. and J.V.V. wrote the paper.

This work was supported by NIH/NIDCD Grants R01DC009994 and R01DC011286. We thank Ryan M. Carey for kindly sharing the temporal sigmoid-fitting Matlab code and Drs. B. A. Johnson and M. Leon for access to and discussion of their raw data files for Matlab evaluation. We thank the John B. Pierce Laboratory machine shop (John Buckley, Michael Fritz, Ron Goodman, Tom D'Alessandro, and Angelo DiRubba) for their excellent technical support. We thank Larry Marks, David Willhite, Michelle Rebello, and Tom McTavish for valuable feedback on an earlier version of the manuscript.

Correspondence should be addressed to Justus V. Verhagen, The John B. Pierce Laboratory, 290 Congress Avenue, New Haven, CT 06519. E-mail: jverhagen@jbpierce.org.

DOI:10.1523/JNEUROSCI.1413-12.2012

Copyright $\odot 2012$ the authors $\quad 0270-6474 / 12 / 327949-11 \$ 15.00 / 0$
(Ressler et al., 1993; Vassar et al., 1993; Mori et al., 1999). In this study, we addressed this basic question by calcium imaging of presynaptic glomerular terminals from olfactory receptor neurons using a rat model.

Odorant coding primarily involves the transformation of an odorant's molecular features into a rough spatial map of activated glomeruli in the OB. Additionally, the temporal dynamics of the glomerular responses may also contribute to the odor quality coding at the level of the OB (Spors et al., 2006; Wesson et al., 2008; Carey et al., 2009; Junek et al., 2010). Several optical imaging studies of the spatiotemporal glomerular activity reflecting presynaptic responses (Wachowiak and Cohen, 2001; Verhagen et al., 2007; Wesson et al., 2008) and time-integrated combined presynaptic and postsynaptic responses (Xu et al., 2003; Johnson and Leon, 2007) have revealed orthonasal odor coding mechanisms at the OB. However, glomerular responses to the retronasal stimulation remain unexplored. In this study, using optical calcium imaging of presynaptic dorsal $O B$ responses to a variety of odorants, we tested the hypothesis that distinct spatiotemporal glomerular activity patterns exist for orthonasal versus retronasal routes of the same odorant. We speculate that these differences may contribute to the distinct cortical responses observed in humans.

Our hypothesis is based on reports supporting a chromatographic model of olfactory epithelium, which suggests that odor flow parameters (Mozell et al., 1984) and odorant distribution across the olfactory epithelium (Scott-Johnson et al., 2000) can influence ORN responses. Indeed, route seems to influence the air-flow pattern inside the nasal cavity (Zhao et al., 2004, 2006), as well as the olfactory population response to odorants at the 
level of the ORNs in vivo in rats (Scott et al., 2007). Here we show that route also influences the spatiotemporal glomerular activity patterns at the $\mathrm{OB}$, and that this effect depends on specific odorant properties, such as polarity and volatility. Since retronasal smell is an essential element of flavor, the present study expands our understanding of the neural bases of flavor perception.

\section{Materials and Methods}

Subjects

Long-Evans female rats weighing 180-200 g were purchased from Charles River Laboratories and housed individually in an environment of controlled humidity (60\%) and temperature $\left(23^{\circ} \mathrm{C}\right)$. The vivarium was set with $12 \mathrm{~h}$ light-dark cycles and all the experiments were performed in the light phase. All the animals were treated according to the guidelines established by the U.S. National Institutes of Health (1986), and the experimental protocols were approved by the Institutional Animal Care and Use Committee of the John B. Pierce Laboratory. Data acquired from nine rats are presented here.

\section{Labeling olfactory receptor neurons}

Olfactory receptor neurons in the dorsal recess of the nasal cavity were loaded bilaterally with dextran-conjugated calcium-sensitive dye (Oregon Green BAPTA 488-1 dextran; Invitrogen) using a well established protocol (Wachowiak and Cohen, 2001) adapted for rats (Verhagen et al., 2007). Animals were held 8-12 d before recording.

\section{Optical window and double} tracheotomy surgery

Before imaging, the dye-infused rats were anesthetized with urethane $(1.5 \mathrm{~g} / \mathrm{kg}$, i.p.), the bone overlying the dorsal surface of the bulb was exposed, thinned, and coated with cyanoacrylate glue to make the bone transparent (Bozza et al., 2004). A double tracheotomy surgery was performed, allowing for the rat to sniff artificially. A Teflon tube (OD $2.1 \mathrm{~mm}$, upper tracheotomy tube) was inserted $10 \mathrm{~mm}$ into the nasopharynx to assure that airflow was restricted to the nose (the epiglottis could otherwise leak air flow via the oral cavity). Another Teflon tube (OD $2.3 \mathrm{~mm}$, lower tracheotomy tube) was inserted into the caudal end of the tracheal cut. Both tubes were fixed and sealed to the tissues using surgical thread and cyanoacrylate glue. The head was stabilized by gluing it to a bar mounted on a stereotaxic head holder designed not to interfere with tracheal breathing. A custom-made Teflon nosemask with minimal dead-space was applied to the clean-shaved nose for orthonasal odor delivery. The upper tracheotomy tube inserted into the nasopharynx was used to deliver odors retronasally (Fig. 1). Local anesthetic (2\% Lidocaine) was applied at all pressure points and incisions. Artificial sniffing was synchronized to the start of each trial. Throughout the surgery and optical recordings, rats' core body temperature was maintained at $37^{\circ} \mathrm{C}$ with a thermostatically controlled heating pad (Omega Engineering).

\section{Optical recordings}

Optical calcium signals from the dorsal OB were recorded using a CCD camera (Redshirt Imaging) with $256 \times 256$ pixel resolution and a frame rate of $25 \mathrm{~Hz}$. This resolution was sufficient to identify single glomeruli at magnifications low enough to image across the entire dorsal and lateral surface of the bulb. The epifluorescence macroscope used was a custommade tandem-lens type (Ratzlaff and Grinvald, 1991) with $\sim 2 \times$ magni-
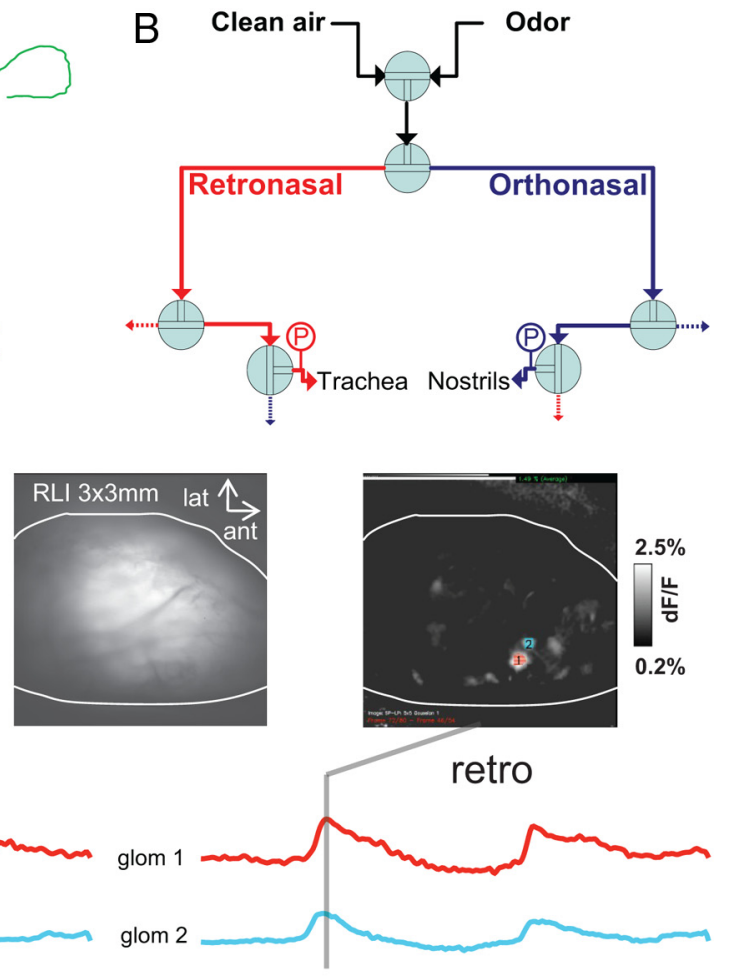

glom 2

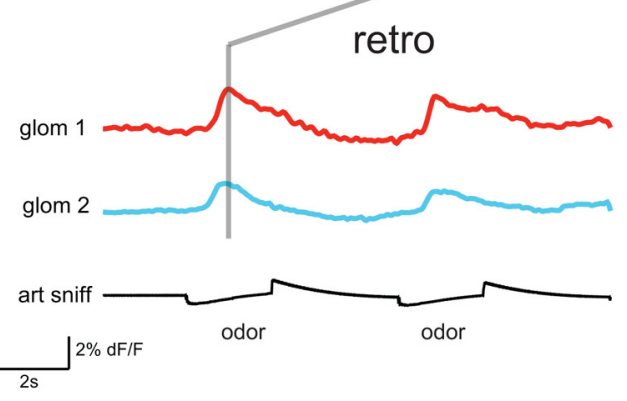

odor

Figure 1. Calcium imaging of the $O B$ glomerular responses to orthonasal and retronasal odorant stimulations. $A, B$, Experimenlines indicate boundaries of the optical window over the left OB. D orthonasal (left) and retronasal (right) glomerular (glom) traces dicated as 1 and 2 on the odor maps. art. sniff, Artificial sniff.

fication and high numerical aperture (0.85-0.95) CCTV objectives for high signal-to-noise ratio. A high-power LED (Luxeon LXHL-PE09, Philips Lumileds) driven by a linear DC power supply acted as the light source. A custom-made DC amplifier (based on a linear Apex power operational amplifier; Cirrus Logic) powered a peltier (OT2.0-31-F1; Melcor) device onto which the LED was glued. The LED-cooling peltier current was proportional to the LED current, yielding a stable illumination. The fluorescence filter set used was FF01-475/50-50 (excitation filter), LP515 (dichroic), and LP530 (emission filter; Semrock). This system provided fast imaging capabilities, a large field of view, and low noise. Raw images were converted to images representing the relative change in fluorescence $(\% \Delta F / F)$ in each pixel and frame after stimulus application. Data analysis was performed using NeuroPlex software (RedShirtImaging) and routines were written in Matlab (version 7.11.0, MathWorks).

\section{Orthonasal and retronasal odorant presentation and odorants}

The schematics of the experimental setup and examples of orthonasal and retronasal imaging trials are shown in Figure 1. We used a novel bidirectional artificial sniffing paradigm with tightly sealed orthonasal nose mask and retronasal odor tube in double-tracheotomized rats (Fig. $1 A, B)$. This closed bidirectional positive-pressure artificial sniffing paradigm, also connected to a pressure sensor ( part 24PCAFA6G; Honeywell) to measure the flow-resistance along both the routes enabled us to properly control the delivery of the odorant stimuli orthonasal as well as retronasally. The time to fill the dead volume was $\sim 120 \mathrm{~ms}$ for either route. The orthonasal stimulus delivery occurred $\sim 2.4 \mathrm{~ms}$ earlier than retronasal delivery (based on $0.01 \mathrm{ml}$ difference in dead-volume and a 
Table 1. Odorants known to activate dorsal $\mathrm{OB}$ in rats and some of their physicochemical properties

\begin{tabular}{|c|c|c|c|c|c|c|c|c|}
\hline Polarity category & Odorant & Abbreviation & $\mathrm{P}\left(\log K_{\mathrm{ow}}\right)^{*}$ & $\mathrm{VP}(\mathrm{mmHg})$ & MV & Density ${ }^{\#}$ & Polar surface $^{\#}$ & $\mathrm{bp}\left({ }^{\circ} \mathrm{C}\right)^{\#}$ \\
\hline Highly polar & 2-hexanone & 2 hex & 1.24 & 11.60 & 100.2 & 0.81 & 17.1 & 127 \\
\hline \multirow[t]{2}{*}{ Polar } & Ethyl butyrate & $E B$ & 1.85 & 12.8 & 116.2 & 0.87 & 26.3 & 120 \\
\hline & Methyl valerate & MV & 1.85 & 19.10 & 116.2 & 0.89 & 26.3 & 126 \\
\hline \multirow[t]{2}{*}{ Nonpolar } & Amyl acetate & AA & 2.26 & 5.60 & 130.2 & 0.87 & 26.3 & 142 \\
\hline & o-tolualdehyde & tolu & 2.26 & 0.36 & 120.2 & 1.04 & 17.1 & 200 \\
\hline Highly nonpolar & Vinyl cyclohexane & VC & 3.95 & 13.40 & 110.2 & 0.80 & 0 & 128 \\
\hline
\end{tabular}

*0ctanol:water partition coefficient, EPI estimate; " ${ }^{*}$ ww.chemexper.com.

Table 2. Summary of MANOVA showing factors affecting glomerular response pattern and interaction between factors ( $p$ value)

\begin{tabular}{|c|c|c|c|c|c|c|c|c|c|c|}
\hline Factor & Rat 1 & Rat 2 & Rat 3 & Rat 4 & Rat 5 & Rat 6 & Rat 7 & Rat 8 & Rat 9 & Percentage of rats \\
\hline Route & $<0.05$ & $<0.01$ & $<0.05$ & $<0.001$ & $<0.001$ & $<0.001$ & $<0.01$ & $<0.001$ & $<0.01$ & $100 \%$ \\
\hline Odor & $<0.001$ & $<0.001$ & $<0.001$ & $<0.001$ & $<0.001$ & $<0.001$ & $<0.001$ & $<0.001$ & $<0.001$ & $100 \%$ \\
\hline Route $\times$ odor & $<0.001$ & $<0.001$ & $<0.001$ & $<0.001$ & $<0.001$ & $<0.001$ & $<0.05$ & $<0.001$ & $<0.001$ & $100 \%$ \\
\hline
\end{tabular}

flow rate time of $240 \mathrm{~ms} / \mathrm{ml}$ ). We chose $250 \mathrm{ml} / \mathrm{min}$ as this was found by Youngentob et al. (1987) to be the average flow rate of inspiratory and expiratory sniffing by awake behaving rats (1.9-8.9 ml/s; their Table 2). The nose-mask guided the air out of the nasal cavity to the exhaust vent during retronasal stimulation, and guided the air into the nasal cavity from the olfactometer during orthonasal stimulation. The Teflon valves (NResearch) involved in this paradigm were fully automated by a program written in LabVIEW (National Instruments). The stimulation paradigm for both the routes was identical and consisted of two $2 \mathrm{~s}$ artificial sniffs of an odorant at an interval of $3 \mathrm{~s}$. All results are based on the responses to the first odor pulse only.

Each imaging session consisted of 80 to 140 trials triggered manually with an intertrial interval of $>3 \mathrm{~min}$. The order of stimulus presentation was randomly permuted for each route and odorant. In each trial, odorants were presented at $250 \mathrm{ml} / \mathrm{min}$ flow-rate as two $2 \mathrm{~s}$ pulses separated by $3 \mathrm{~s}$ interval (Fig. $1 C, D$ ) using a custom-built multichannel autoswitching flow dilution olfactometer (Lam et al., 2000) with dedicated lines for each odorant to avoid cross-contamination. This allowed for the continuous control of odorant concentration over $1.5 \mathrm{log}$ units. After each stimulus, the nasal cavity was flushed with clean humidified (sparging distilled water) air for $1 \mathrm{~min}$. The olfactometer output was routed to a set of route-switching valves that were mounted on the side of the stereotax so as to minimize the dead space. Odorant concentrations are indicated as percentage saturated vapor (\% s.v.). Medical-grade air was used to dilute the vapor in the headspace of odorant reservoirs to generate the desired concentration. Odorants were diluted before reaching common tubing to maximize purity. The odor manifolds were automatically flushed with clean air after each stimulation cycle. Our system allowed precise control of the odorant access to the nasal cavity both orthonasal and retronasally. With this paradigm, we were able to control intranasal odorant concentration as well as air flow rates. Monomolecular odorants were chosen from the family of odorants whose effects on the dorsal bulb have been previously characterized (Johnson and Leon, 2000; Uchida et al., 2000; Meister and Bonhoeffer, 2001; Wachowiak and Cohen, 2001). In collaboration with Drs. Johnson and Leon, we identified 16 odorants that are most selective for the dorsal bulb based on quantitative MATLAB analyses of their raw 2-DG datasets. These odorants with their partition coefficients and other parameters (EPI estimates) are shown in Table 1. The entire odorant delivery system was made of Teflon. All the odorants were obtained from Sigma-Aldrich and stored under nitrogen in the dark.

\section{Measurement of breathing}

Breathing was measured as the movement of the thorax by a piezoelectric strap around the animal's chest. During each respiration cycle, one sharp upward deflection in the piezoelectric signal occurred during thorax expansion (inspiration). The point of onset of this deflection occurring before and after the stimulus onset time was used as a time reference for estimating instantaneous breathing frequency and assessing occurrence of response coupling with breathing cycle. The temporal parameters were measured in reference to the stimulus onset time recorded directly by a pressure sensor connected to the bidirectional artificial sniffing setup.

\section{Data analysis}

Identification of activated glomeruli. Datasets consisting of optical images of $256 \times 256$ pixels sampled at $25 \mathrm{~Hz}$, pressure signals sampled at $200 \mathrm{~Hz}$, breathing signals, information on odor identity, odor concentration, and flow rate were acquired using Neuroplex software on a $12 \mathrm{~s}$ trial-by-trial basis (Fig. $1 C, D$ ). Script files written in MATLAB were used to extract and preprocess these data to correct global noise in every imaged frame. The preprocessed images were then averaged across trials for each stimulus to identify regions of interest (ROI; activated glomeruli). Focal changes in fluorescence in the OB have been shown previously to correspond to individual glomeruli (Belluscio and Katz, 2001; Meister and Bonhoeffer, 2001; Wachowiak et al., 2004).

Estimation of response magnitude. Using the identified ROI, we then extracted average glomerular response curves ( $F$ traces) based on stimulus onset times. These $F$ traces guided the selection of optimum preframe (before stimulus onset) and postframe (about max response) windows, which consisted of 15-21 frames (600-840 ms). The response magnitudes across each ROI were then measured using this window for each trial as percentage change in fluorescence before and after stimulus onset (\% $\Delta F / F)$, as reported previously (Verhagen et al., 2007). MANOVA (odorant $X$ route) was then performed across all trials from an animal (Table 2) and any ROI for which effect of odorant (including odorless air, delivered via a separate clean line) was not significant was removed from further analyses.

Spatial analysis of orthonasal versus retronasal response patterns. Peak response amplitudes $(\% \Delta F / F)$ at the ROIs were compared between routes of stimulation across odorants. Correlation analysis and ANOVA were used to establish whether changing the route of stimulation changes the spatial odor map, and how the orthonasal versus retronasal differences were affected by physicochemical properties of the odorants. Multidimensional scaling was performed in Systat (v10.2, method: Kruskal, mono). Averages are reported $\pm \operatorname{SEM}(\mathrm{SD} / \sqrt{ } n)$. Alpha level was set at 0.05 .

Temporal analysis of orthonasal versus retronasal response patterns. To measure temporal parameters of the glomerular response, a custom algorithm was developed that fitted the optical signals from each ROI to a double sigmoid function, as described previously (Wesson et al., 2008; Carey et al., 2009). The analysis allowed robust and objective measurement of response timing. Briefly, the signal from each ROI was bandpass filtered (second-order Butterworth, $0.4-8 \mathrm{~Hz}$ ) followed by fourth-order Daubechies wavelet decomposition, soft thresholding of the coefficients at level 3 , and then reconstruction. The onset time was defined based on the time of peak in the product of the first and the second derivatives of the optical signal. Starting at this time, each response was fitted (leastsquares curve fitting) with a double-sigmoid function (a sigmoid rise followed by a sigmoid fall). The time of the peak of this response was 
defined as the peak in this fitted response function, rather than the peak of the raw optical signal.

To compare the temporal dynamics of orthonasal versus retronasal glomerular responses, we extracted parameters of the time course of responses at each glomerulus. The parameters were first averaged across glomeruli for each odorant and route separately. Then the mean for each odorant per route was averaged across nine rats. The temporal delay for the retronasal response relative to the corresponding orthonasal response is referred to as "retronasal delay" or "retro delay" in this paper.

Correlation analysis and ANOVA were used to establish whether the route of stimulation affected the temporal parameters and how the orthonasal versus retronasal differences were affected by physicochemical properties of the odorants.

Measurement of temporal relationship between glomerular responses and breathing. Our stimulus onset time was not triggered from the rats breathing cycle or other signals, but instead was intended to be randomly initiated. This could have biased our results, in the unlikely case where potential breathing-related blood oxygenation level-, blood volume-, or blood flow rate-related noise was not randomly distributed between orthonasal and retronasal trials. We recorded breathing signals from each experimental animal throughout the experiments. Using a Matlab script, we determined points of interest relative to the inspiration just before the stimulus onset (first inspiration) and extracted the values from each individual trial based on the instantaneous breathing frequency at the time of stimulus onset. We then determined whether peak response for each route of stimulation corresponded to certain phase of breathing. We also compared orthonasal and retronasal breathing rate, stimulus onset time from the preceding inspiration, position of stimulus onset within a breath cycle (range: $0-1$ ), peak response time from the preceding inspiration, and number of breath cycles until peak response.

\section{Results}

Orthonasal and retronasal glomerular response patterns largely overlap

We first investigated how similar the retronasal and orthonasal glomerular response patterns are. Figure 2 shows an example of averaged $(n=3)$ odor maps in the OB at their peak response for orthonasal 4\% 2-hexanone (2hex), orthonasal 4\% methyl valerate (MV), and retronasal 4\% MV. Responses of glomerulus 1 and 12 vary strongly across the three conditions. Glomerulus 1 responded mainly to orthonasal MV, much less to the retronasal stimulation thereof, and not at all to orthonasal $2 \mathrm{hex}$, whereas glomerulus 12 responded strongest to orthonasal $2 \mathrm{hex}$. The average responses (mean \pm SEM) for these three stimuli at 16 glomeruli appear in Figure $2 E$. Paired $t$ test between orthonasal 2 hex and orthonasal MV responses revealed significant differences at nine glomeruli. Between orthonasal and retronasal MV, however, responses were different significantly only at two glomeruli. Correlation analysis of the responses between orthonasal MV and orthonasal 2 hex across all 16 glomeruli showed a negative correlation $(r=-0.61)$, while that between orthonasal versus retronasal MV exhibited a strong positive correlation $(r=0.74)$.

Across all nine rats for six odors and air, the glomerular pattern correlation between routes was $0.38 \pm 0.09$ (mean \pm SEM; $n=7)$, which increased to $0.45 \pm 01(n=6)$ after removing putative outlier tolualdehyde $(r=-0.03)$. The pattern similarity between different odors presented via the same route was $0.27 \pm$ 0.03 ( $n=42$ odor-odor combinations), which decreased to $0.22 \pm 0.04(n=30)$ after removing tolualdehyde. Response patterns were more similar for the same odorants between routes than for the same route between odors $(p<0.05)$, but only after removing tolualdehyde, the retronasal responses of which did not relate to the orthonasal ones. This was substantiated by using multidimensional scaling (Fig. $3 B$ ) to explore the response pattern similarities across all stimuli at once. Stimulus pairs are linked by lines. Figure $3 B$ shows that odors are clearly differenti- ated along the hypothetical odorant axis, and are also differentiated (but to a lesser degree) along the route axis. The dotted line separates five of the six odorants by route.

When we analyzed the similarity in response magnitudes between all orthonasal and retronasal pairs in a single correlation, it yielded an even higher correlation of $r=0.51$ (893 pairs; Fig. $2 G$ ). We therefore conclude that orthonasal versus retronasal $\mathrm{OB}$ response patterns elicited by the same odorant differ less than response patterns elicited by two different odorants via the same route. In other words, odor route affects response patterns less than odorant identity.

To estimate the degree of overlap between orthonasal and retronasal odor maps, we arbitrarily set $<0.05 \% \Delta F / F$ as noise and calculated for each stimulus the percentage of the total number of responsive glomeruli in an animal that was responsive to each route. On average ( 6 odorants, 9 rats, 779 glomerular responses), we found $69 \pm 9 \%$ (mean \pm SD) overlap between the two routes (Fig. $2 F$ ). The remaining $20 \pm 4 \%$ and $11 \pm 6 \%$ glomeruli were activated, respectively, by orthonasal and retronasal stimulation only. In terms of the number of glomeruli imaged per rat, $11.14 \pm 2.37$ (mean $\pm \mathrm{SD}$ ) responded to both routes while $2.48 \pm 0.48$ and $1.51 \pm 0.73$ glomeruli responded only to orthonasal and retronasal stimulation, respectively.

\section{Retronasal response amplitudes are smaller than orthonasal ones}

We next asked how response magnitudes were affected by the routes of odorant delivery. Figure $3 A$ compares retronasal response magnitudes to the corresponding orthonasal responses across six odorants and clean air, ordered by decreasing polarity (octanol: water partition coefficient, $k_{\mathrm{ow}}$ ). Figure $3 \mathrm{~A}$ shows both the mean glomerular response (first $\% \Delta F / F$ averaged across glomeruli in each rat and then averaged across rats, mean \pm SEM; $n=5 \sim 9$ rats) for each route and, the retronasal/orthonasal (r/o) ratio of $\% \Delta F / F$ (averaged across rats; mean \pm SEM; $n=5 \sim 9$ ) providing an estimate of the pairwise retronasal response efficacy relative to the orthonasal response. Responses to the orthonasal 2hex $(0.47 \pm 0.05 \% \Delta F / F)$ were significantly larger than the retronasal responses $(0.27 \pm 0.10 \% \Delta F / F ; p<0.05, \mathrm{r} / \mathrm{o}=0.52 \pm$ $0.19, n=6)$. Ethyl butyrate, showed relatively larger responses both orthonasally $(0.65 \pm 0.07 \% \Delta F / F)$ and retronasally $(0.37 \pm$ $0.09 \% \Delta F / F)$, and the difference was significant $(p<0.05, \mathrm{r} / \mathrm{o}=$ $0.59 \pm 0.14, n=9)$. MV responses were also robust orthonasally $(0.60 \pm 0.08 \% \Delta F / F)$ but less so retronasally $(0.30 \pm 0.08 \% \Delta F /$ $F)$, showing a significant difference $(p<0.05, \mathrm{r} / \mathrm{o}=0.61 \pm 0.18$, $n=8)$. Amyl acetate showed a significantly smaller $(p<0.05)$ retronasal response $(0.14 \pm 0.06 \% \Delta F / F)$ compared with an almost double orthonasal response $(0.31 \pm 0.04 \% \Delta F / F, r / o=$ $0.49 \pm 0.18)$. Tolualdehyde retronasal responses $(0.10 \pm$ $0.05 \% \Delta F / F)$ were also the smallest among the six odorants tested, and the orthonasal responses $(0.26 \pm 0.07 \% \Delta F / F)$ were significantly larger $(p<0.05, \mathrm{r} / \mathrm{o}=0.34 \pm 0.24, n=5)$. Vinyl cyclohexane, however, was only close to a significant difference in the response between the routes (orthonasal: $0.19 \pm 0.05 \% \Delta F / F$, retronasal: $0.11 \pm 0.04 \% \Delta F / F, p=0.07, \mathrm{r} / \mathrm{o}=0.62 \pm 0.20, n=$ $7)$. By contrast, clean air evoked responses of similar magnitude (orthonasal: $0.16 \pm 0.03 \% \Delta F / F$, retronasal: $0.13 \pm 0.05 \% \Delta F / F$, $p=0.22, \mathrm{r} / \mathrm{o}=0.71 \pm 0.36, n=9)$. Across all orthonasal versus retronasal responses, the orthonasal responses $(0.39 \pm$ $0.04 \% \Delta F / F)$ were significantly larger $(p<0.01, \mathrm{r} / \mathrm{o}=0.63 \pm$ $0.12, n=9)$ than the retronasal responses $(0.23 \pm 0.04 \% \Delta F / F)$. The correlation analysis across all 893 orthonasal-retronasal stimulus pairs, shown in Figure $2 G$, similarly suggests that retro- 


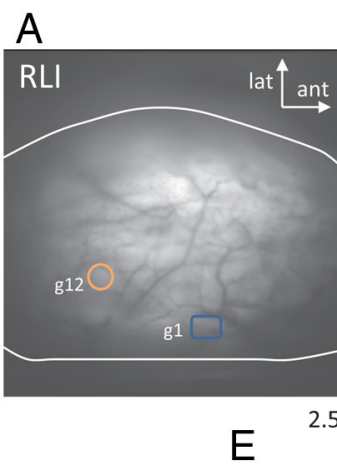

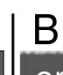

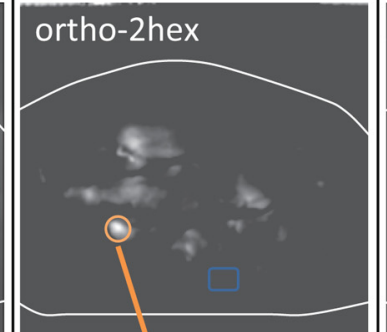

E 2.5
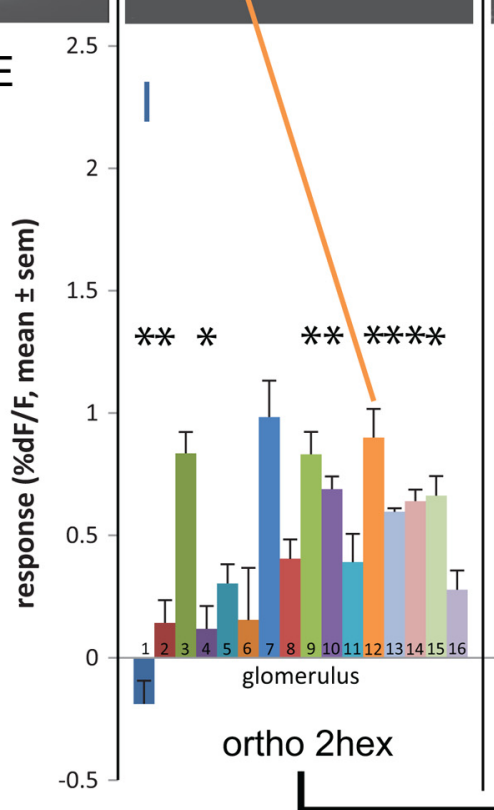

C
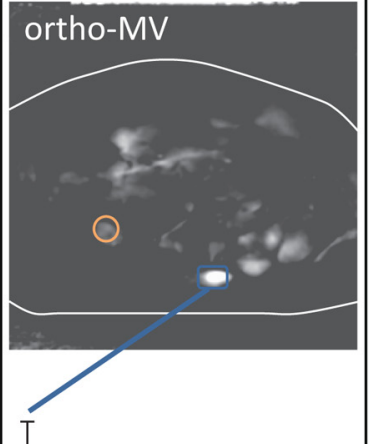

(1)

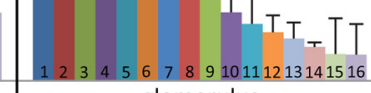

glomerulus

$r=-0.61$

$\mathrm{F}$

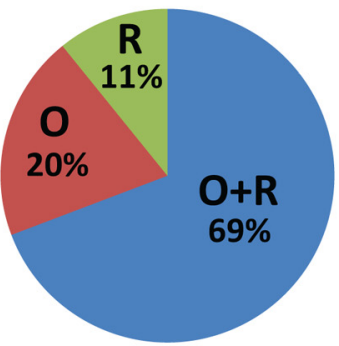

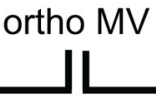

ortho MV
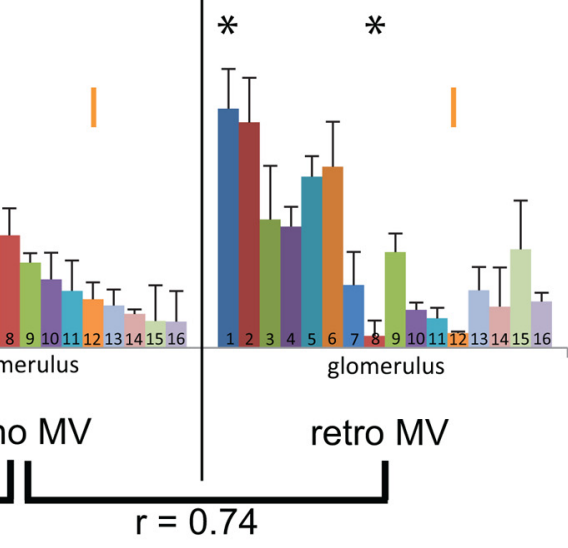

glomerulus

\section{retro MV}

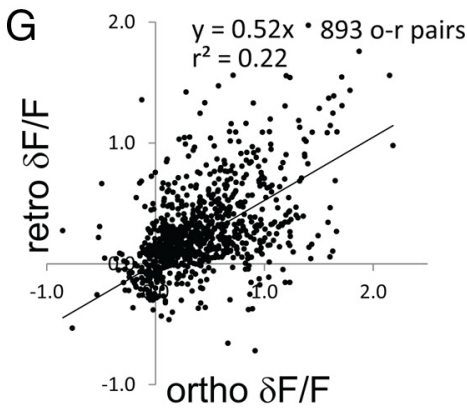

Figure 2. Orthonasal and retronasal glomerular response patterns elicited by the same odor overlap but differ in terms of response size. $A-E$, An example from a rat. $A$, Resting light intensity (RLI) image of the left bulb. $\boldsymbol{B}-\boldsymbol{D}$, Averaged $(n=3)$ odor maps for orthonasal $4 \% 2$ hex $(\boldsymbol{B})$, orthonasal $4 \% \mathrm{MV}(\boldsymbol{C})$, and retronasal $4 \% \mathrm{MV}(\boldsymbol{D})$. Positions of two of the activated glomeruli ( $\mathrm{g} 1$ and g12) are marked by the orange circle and blue square on each map. White lines indicate the boundaries of the bulb visible through the optical window. $\boldsymbol{E}$, Bar diagram showing the glomerular response patterns for orthonasal 2 hex versus orthonasal MV, and orthonasal versus retronasal MV. Between orthonasal 2hex and orthonasal MV, the responses of nine glomeruli ( $g 1, g 2, g 4, g 9, g 10$, and $g 12 \sim g 15)$ were significantly different $\left({ }^{*} p<0.05\right)$. Between orthonasal and retronasal $\mathrm{MV}$, however, responses were significantly different only at $\mathrm{g} 1$ and $\mathrm{g} 8\left({ }^{*} p<0.05\right)$. $\boldsymbol{F}, \mathbf{G}, 0 \mathrm{verall}$ picture from nine rats. $F$, The average percentage of glomeruli in a rat responding only orthonasally $(0$; red), retronasally ( $R$; green), or to both routes $(0+R$; blue) (based on noise-level set at $<0.05 \% \Delta F / F)$. G, Scatter plot to show the correlation between all 894 orthonasal-retronasal glomerular response pairs (152 glomeruli, 5-9 rats, 3-7 odorants per rat).

nasal response amplitudes were $52 \%$ of the orthonasal magnitudes, which is close to the $63 \%$ suggested by the ratio above. These results indicate that, in general, retronasal responses to odorant stimuli are significantly smaller than orthonasal responses, despite identical odor concentrations and flow rate.

\section{Volatility enhances retronasal response efficacy}

To determine whether there was a relationship between specific physicochemical properties of the odorants and their retronasal response efficacy relative to orthonasal responses, we plotted the odors and these properties relative to the mean r/o ratio. We found that the relative retronasal efficacy (r/o ratio) did not correlate well with the lipophilicity/partition coefficient $\left[\mathrm{P}\left(\log k_{\mathrm{ow}}\right)\right.$; $r=0.21, p=0.34$; Fig. $4 B$ ] or polar surface area [polar surface $\left(\AA^{2}\right), r=-0.10, p=0.42$; Fig. $\left.4 E\right]$, but did correlate strongly with vapor pressure (mmHg; $r=0.92, p<0.005$, Fig. $4 A$ ), density $(\mathrm{g} / \mathrm{ml} ; r=-0.80, p<0.05$; Fig. $4 C)$, or boiling point $\left({ }^{\circ} \mathrm{C} ; r=\right.$ $-0.93, p<0.005$; Fig. $4 D)$. The orthonasal and retronasal response magnitudes at best only trended toward significant correlations with these properties (data not shown).The orthonasalretro pattern similarity was found to increase only with decreasing boiling point $\left(r^{2}=0.88, n=6, p<0.005\right)$ and with 

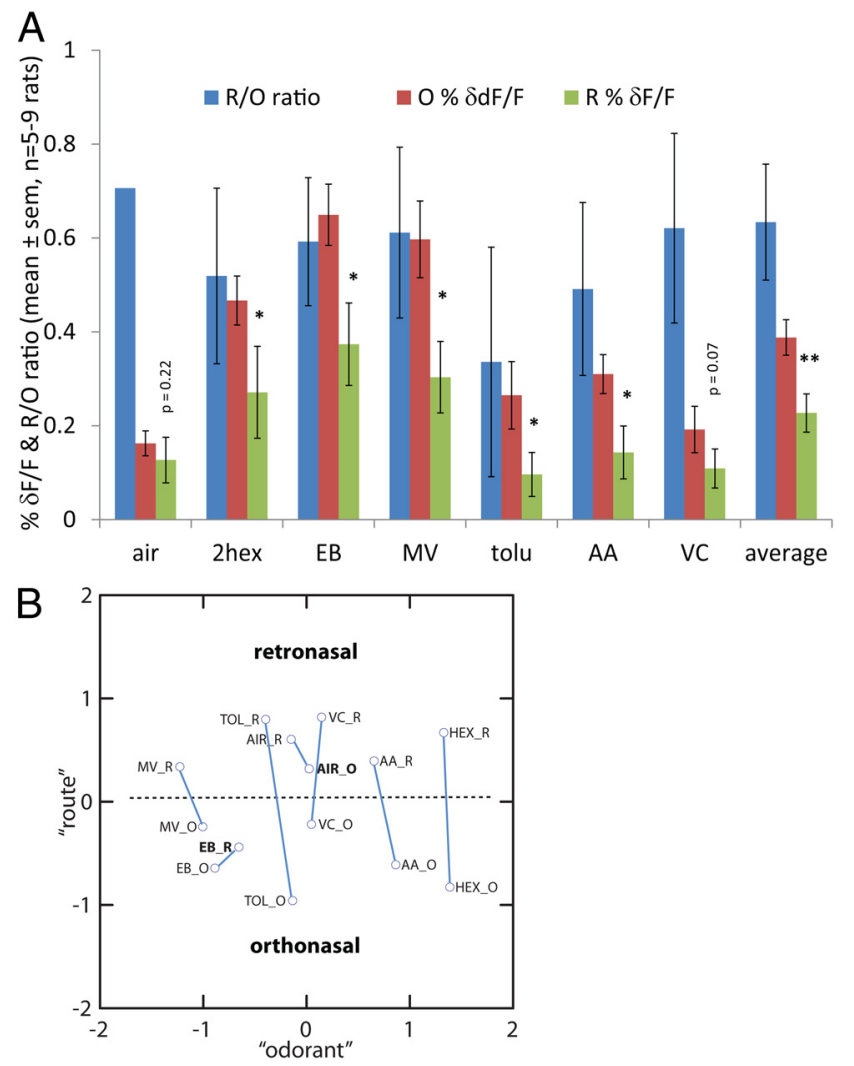

Figure 3. Comparison of orthonasal versus retronasal response magnitudes for different odorants. $A$, The averaged orthonasal and retronasal responses are shown, as well as their ratio to their left (mean $\pm \mathrm{SEM}, n=6 \sim 9$ rats). The magnitude of retronasal responses was significantly smaller than that of the orthonasal ones, except for air and vinyl cyclohexane (VC). Overall, retronasal responses were $63 \%$ of the orthonasal responses. Effect of route: ${ }^{*} p<0.05,{ }^{* *} p<0.01$. EB, Ethyl butyrate; AA, amyl acetate; tolu, 0 -tolualdehyde; TOL, 0 -tolualdehyde. $\boldsymbol{B}$, Multidimensional scaling diagram of response pattern similarities $\left(r^{2}=0.85\right)$ shows a relatively small distance between routes (connected via blue lines, across suggested route axis) compared with between odorants (across the suggested odorant axis). Dotted line roughly separates the two routes with exception of boldfaced ethyl butyrate and air.

decreasing density $\left(r^{2}=0.79, n=6, p<0.01\right)$, and additionally with $\mathrm{r} / \mathrm{o}$ response ratio $\left(r^{2}=0.68, n=6, p<0.05\right)$.

We'd like to point out that these findings were robust and not dependent on a hinge-like effect of the single high-density and high-boiling point odorant (Fig. 4C,D), in that in a complimentary analysis based on correlation-derived slopes rather than ratios (Fig. 3A), the same results were obtained. There we also included two additional odorants: the highly polar cyclohexane and highly nonpolar mesitylene with continuous values for density and boiling point $\left(0.95 \mathrm{mg} / \mathrm{ml}\right.$ and $0.87 \mathrm{mg} / \mathrm{ml}$, and $155^{\circ} \mathrm{C}$ and $164^{\circ} \mathrm{C}$, respectively). In that analysis, the relation between density and $\mathrm{r} / \mathrm{o}$ ratio yielded $r^{2}=0.76(p<0.01)$ and the relation between boiling point and r/o ratio yielded $r^{2}=0.83(p<0.001)$. These two odors were otherwise excluded from analyses due to the low number of rats tested with them (4 rats).

As higher vapor pressure, lower density, and lower boiling point tend to make an odorant more volatile, these results indicate that higher volatility increases especially retronasal efficacy and thereby increases the pattern similarity between the orthonasal and retronasal response in the presynaptic dorsal OB.
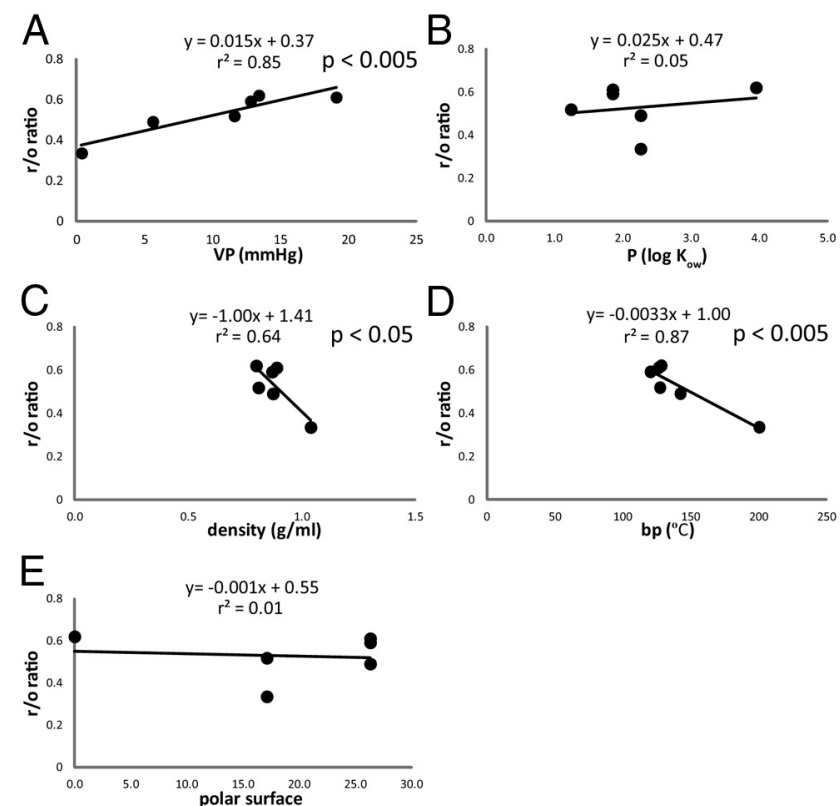

Figure 4. Retronasal relative response efficacy correlates with the volatility of the odorants. $\boldsymbol{A}-\boldsymbol{E}$, The mean $\mathrm{r} / 0$ response ratio of Figure $3 A$ for each odorant is plotted against vapor pressure $(\mathrm{VP}, \mathrm{mmHg} ; \boldsymbol{A})$, partition coefficient $\left[\mathrm{P}\left(\log \mathrm{K}_{\mathrm{oww}}\right) ; \boldsymbol{B}\right]$, density $(\mathrm{g} / \mathrm{ml} ; \boldsymbol{C})$, boiling point $\left(\mathrm{bp},{ }^{\circ} \mathrm{C} ; \boldsymbol{D}\right)$, and polar surface area $\left(\AA^{2} ; \boldsymbol{E}\right)$. Note that the r/o ratio correlates significantly with vapor pressure $(r=0.92, p<0.005)$, density $(r=-0.80, p<0.05)$, and boiling point $(r=-0.93, p<$ $0.005)$, but not with the partition coefficient or polar surface.

\section{Retronasal responses show slower but more consistent temporal dynamics than orthonasal responses}

The temporal dynamics of calcium responses may contain information about odor quality at the level of the OB (Spors et al., 2006; Junek et al., 2010). Significant differences may exist between the response latencies of different glomeruli to the same odorant and, for a given glomerulus, different odorants can evoke responses with different latencies (Spors et al., 2006). To compare the temporal dynamics of orthonasal versus retronasal glomerular responses we examined the time course of the responses of each glomerulus (see Materials and Methods, above; Fig. 5A). The averaged values of each parameter for each odorant (ordered by polarity) and each route are shown separately in Figure 5, $B$ and $C$. Temporal response parameters were relatively variable across orthonasally presented odorants, notably for $\mathrm{t}_{10}\left(F_{(6,50)}=12.1, p<10^{-7}\right.$, one-way ANOVA $), \mathrm{t}_{50}$ $\left(F_{(6,50)}=3.4, p<0.01\right)$ and rise-time $\left(F_{(6,50)}=6.4, p<10^{-4}\right)$. Retronasal dynamics barely varied between odorants, only for $t_{50}$ $\left(F_{(6,50)}=3.3, p<0.01\right)$. This is more clearly shown in Figure $5 E$, which shows the reconstituted sigmoidal plots for orthonasal (red) and retronasal (blue) odorants.

To determine whether any of the temporal parameters differ between the orthonasal and retronasal route, we subtracted retro values from the orthonasal values for each odor and parameter in pairwise fashion (Fig. 5D). We found that the average duration of the lag between the stimulus onset and the response onset (start) was $-439 \pm 30 \mathrm{~ms}$ for orthonasal and $-550 \pm 22 \mathrm{~ms}$ for retronasal responses, with a resulting retronasal delay (orthonasal minus retronasal; retro delay) of $100 \pm 39 \mathrm{~ms}$ relative to the orthonasal response $(p<0.0001, n=57 ; 7$ odors $\times 9$ rats, 6 missing data points; paired two-tailed $t$ test). The retronasal route was associated with slightly faster response from start to $10 \%$ of peak amplitude (retro delay $t_{10}$, $42 \pm 11 \mathrm{~ms} ; p<0.0001$; Fig. $5 D)$, but similar from start to $50 \%\left(t_{50}\right.$, $11 \pm 7 \mathrm{~ms} ; p=0.20$ ). The retronasal responses were consistently slower to reach $90 \%$ of peak $\left(t_{90},-45 \pm 22 \mathrm{~ms} ; p=0.002\right)$ and peak 

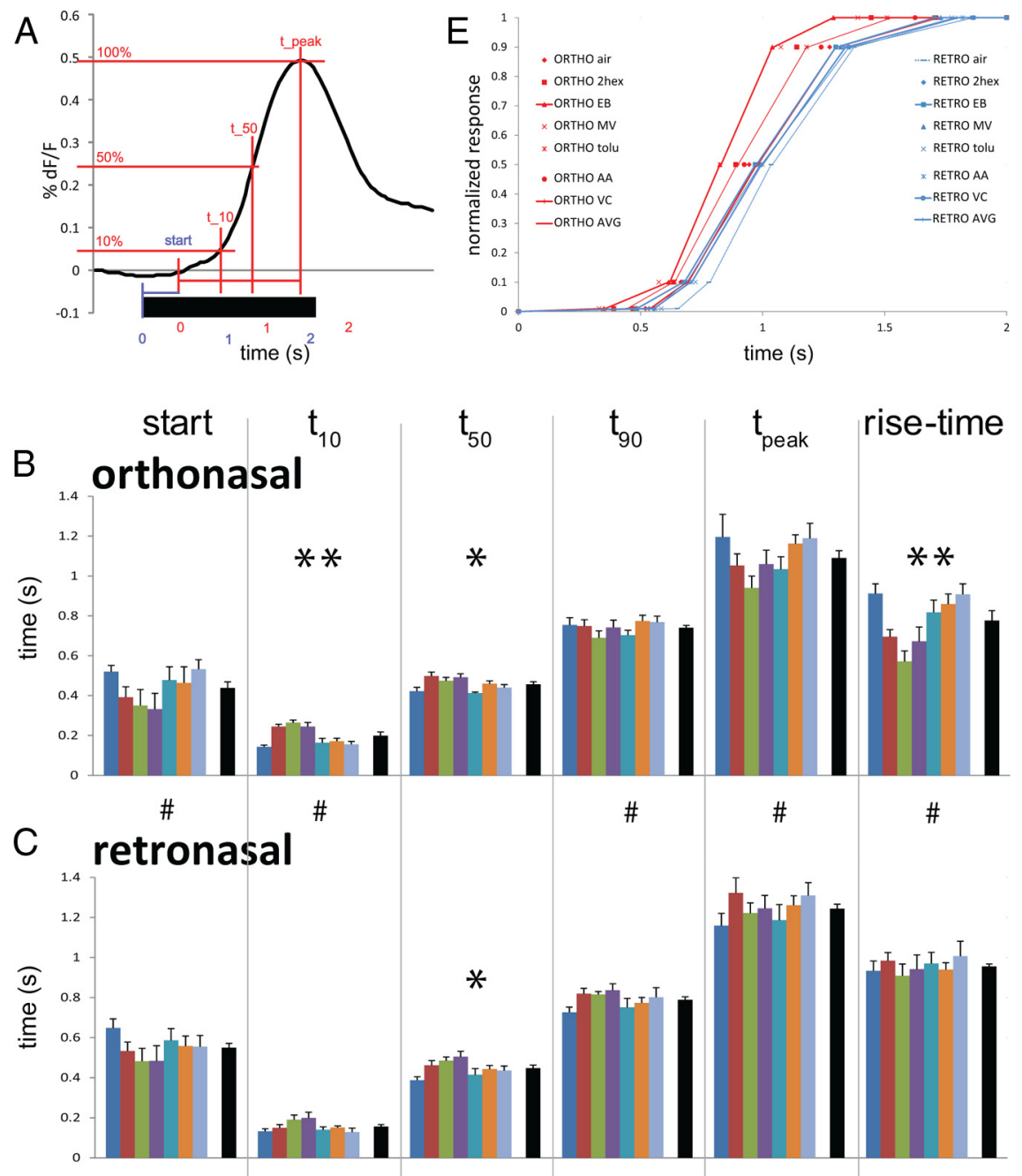

\#
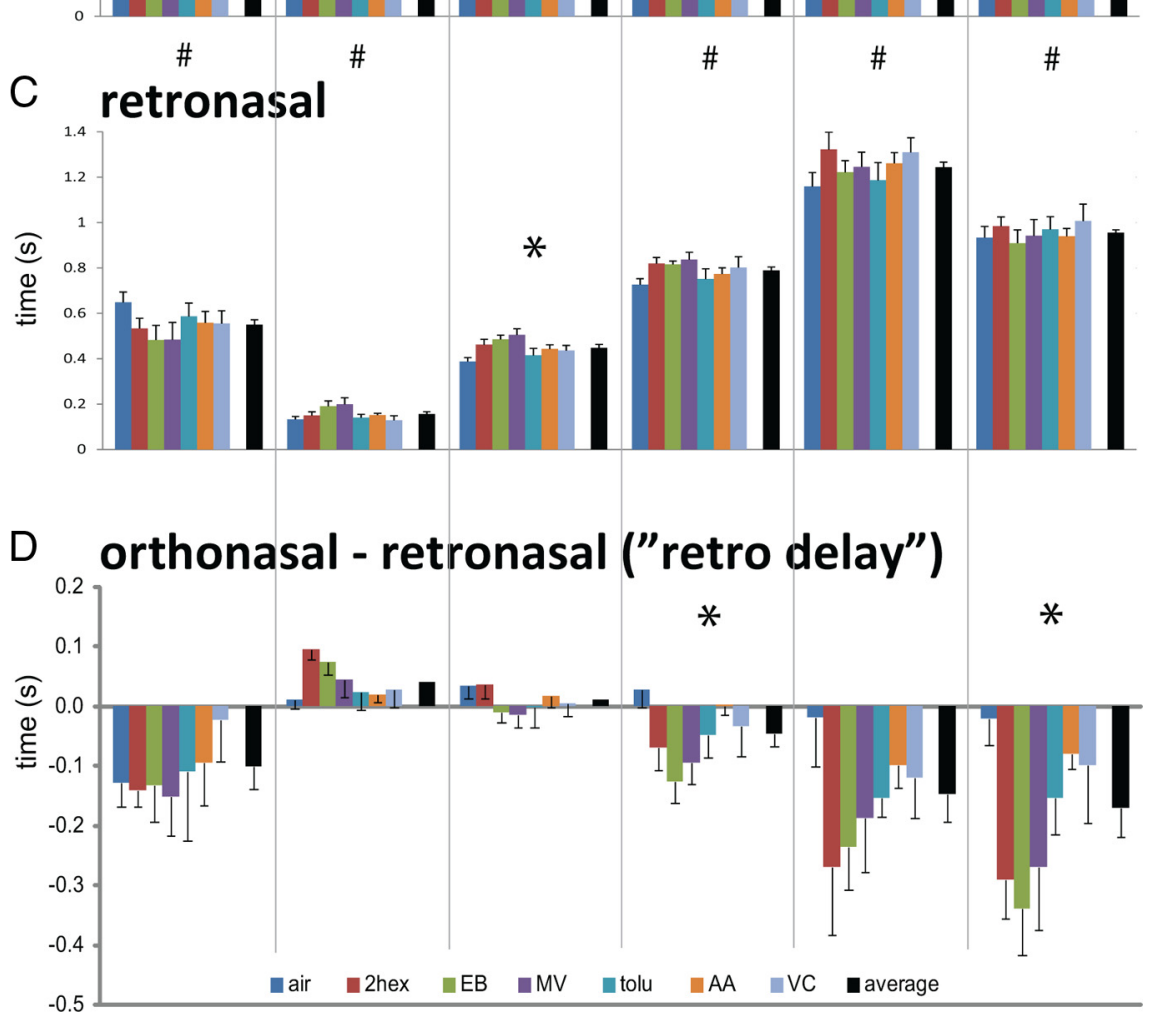

Figure 5. Temporal dynamics of orthonasal versus retronasal odor responses. $\boldsymbol{A}$, Response traces were fitted with a double sigmoidal function and the time of response onset (start), time to $10 \%, 50 \%$, and $90 \%$ of the response peak $\left(t_{10}, t_{50}\right.$, and $\left.t_{90}\right)$, the time of the response peak $\left(t_{\text {peak }}\right)$, and rise time were determined. $\boldsymbol{B}-\boldsymbol{D}$, Descriptors of the time course of orthonasal $(\boldsymbol{B})$ and retronasal $(\boldsymbol{C})$ glomerular responses for each odorant are shown. There was a significant difference between orthonasal and retronasal dynamics, except for $t_{50}$. Dynamics varied more strongly when odors were present orthonasally than retronasally. Based on the same data as used for Figures $3 A$ and 4 ; see Results for further details. Effect of odor: ${ }^{*} p<0.05,{ }^{* *} p<0.001$. Effect of route: ${ }^{\#} p<0.05 . \boldsymbol{E}$, Reconstituted sigmoidal time courses for each odor and route (orthonasal, red; retronasal, blue). For clarity, only the overall average (AVG) and the most extreme time courses are shown with connecting lines: ethyl butyrate (EB), $V($ and the slowest retronasal air. AA, amyl acetate; tolu, o-tolualdehyde; $\mathrm{VC}$, vinyl cyclohexane.

itself $\left(t_{\text {peak }},-147 \pm 47 \mathrm{~ms} ; p=0.0001\right)$. On average, the rise time for retronasal response was longer than that for orthonasal response by $169 \pm 49 \mathrm{~ms}(p<0.0001)$. Unpaired two-sided $t$ tests yielded the same conclusions. rise-time

We also performed two-way ANOVA to understand possible effects of route, odor, and their interactions on each of the temporal parameters. Route showed a significant $\left(F_{(1,100)}>7.6, p<0.01\right)$ effect on each of the temporal parameters except for $t_{50}(p=$ 0.35 ), consistent with the $t$ tests above. Likewise, across both routes, odor had an effect $\left(F_{(6,100)}>2.4, p<0.05\right)$ on $t_{\text {onset }}, t_{10}, t_{50}$, and rise time, but not on $t_{90}(p=0.41)$ or $t_{\text {peak }}$ $(p=0.20)$. The interaction between route and odor was significant only for the rise time $\left(F_{(6,100)}=2.5, p<0.05\right)$. A one-way ANOVA of odor effect on retro delay only showed an effect on rise time $\left(F_{(5,60)}=\right.$ 2.9, $p<0.05)$, as expected based on the two-way ANOVA, and also on $t_{90}\left(F_{(5,60)}=\right.$ 2.3, $p<0.05$ ).

It should be noted that the response latencies we report are on the long side, but are not outside the bounds of prior measures. Response onset delay was $\sim 0.5 \mathrm{~s}$, of which $\sim 120$ ms was due to dead space in the olfactometer (see Materials and Methods, above). The remaining $\sim 380 \mathrm{~ms}$ are not unusual, as similar delays have been reported before using EOG. For example, Scott's (2006) Figure 3, which shows the ventrolateral response to isoamyl acetate at $200 \mathrm{ml} /$ min flow rate takes $300-400 \mathrm{~ms}$ to develop.

We also would like to point out that, despite having minimized the difference of the dead space of our olfactometer between both routes, it is possible that within the rat itself differences in dead space remain (i.e., between the odor mask or retronasal canula and olfactory epithelium). These could account for the difference in onset delay between routes (Fig. 5D,E), but does not explain the effect of route or polarity on the other parameters from which this delay was subtracted $\left(t_{10}, t_{50}, t_{90}, t_{\text {peak }}\right.$, and rise time; Figs. 5, 6). Indeed, we subtract onset delay from the other parameters and variability remains (Fig. $5 B, C$, pound signs), as tested by ANOVA.

Overall, these observations suggest that the time course of retronasal responses is slower than that of orthonasal responses, including a longer onset latency, longer time to peak, and longer rise time. Moreover, the response dynamics varied less across odors presented retronasally than orthonasally.

\section{Retronasal delay for the onset, time to peak, and rise time varies with the polarity of the odorants}

We then asked if and how retronasal delays of Figure $5 D$ correlated with specific odorant properties (Table 3). As shown in Figure 6 $A$, mean retro delay (the values of which are the inverted values of Fig. $5 D$ for clarity) for the onset of the response for each odorant was plotted against its 

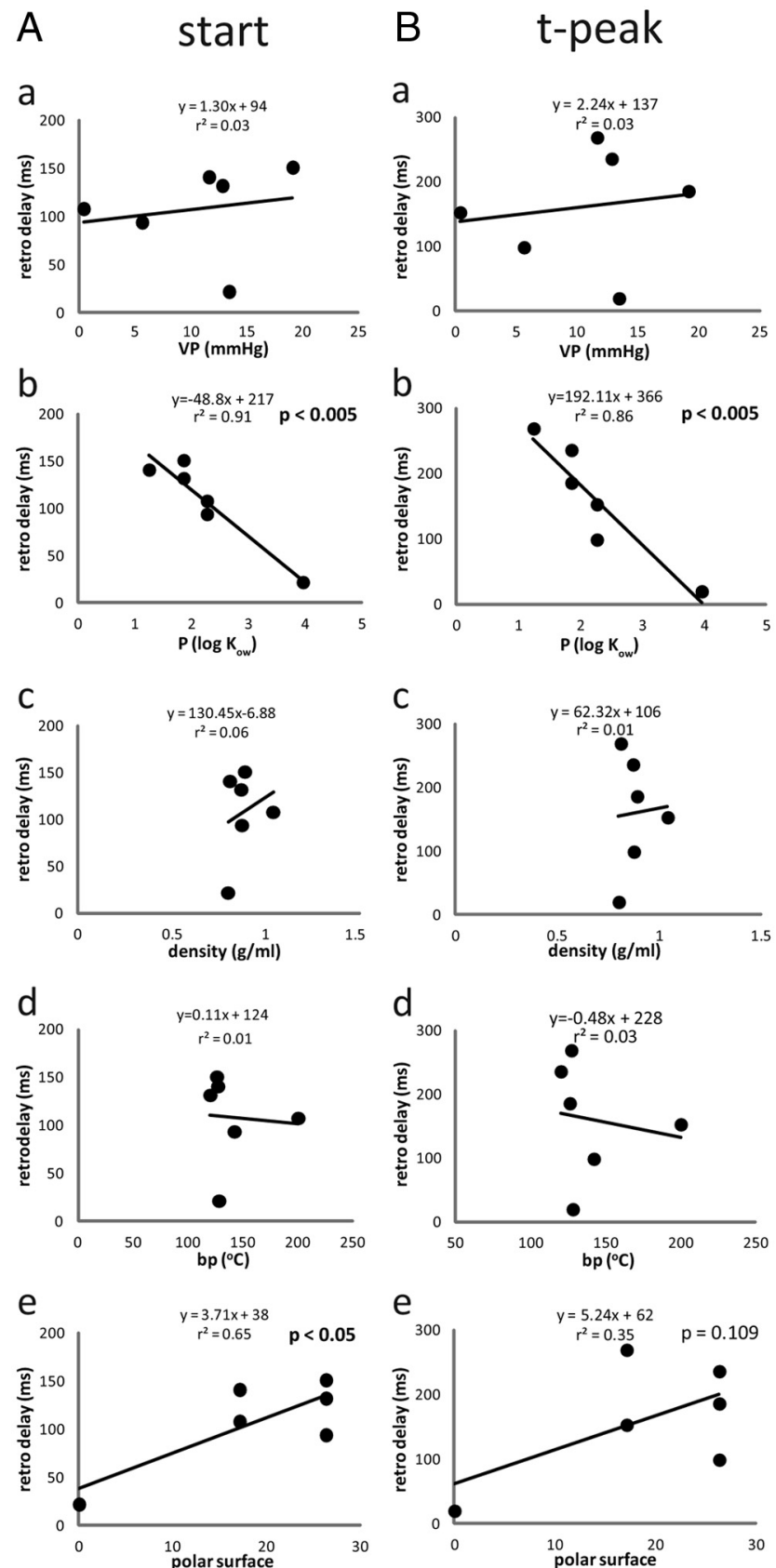

Figure 6. Retronasal delay (inverted values from Fig. 5D for clarity) for the onset of the response and time to peak correlates with the polarity of odorants. $A$, Mean retro delay for the onset of the response for each odorant is plotted against vapor pressure (VP; $\boldsymbol{a}$ ), partition coefficient $(P ; \boldsymbol{b})$, density $(\boldsymbol{c})$, boiling point $(b p ; \boldsymbol{d})$, and polar surface $(\boldsymbol{e})$. Note that the retro onset delay does not correlate with vapor pressure and density, but does with polar surface $(r=0.81, p<0.05)$ and $\mathrm{P}(r=-0.95, p=0.005)$. $\boldsymbol{B}$, Mean retro delay for $t_{\text {peak }}$ is plotted against vapor pressure $(\boldsymbol{a}), \mathrm{P}(\boldsymbol{b})$, density $(\boldsymbol{c})$, boiling point $(\boldsymbol{d})$, and polar surface $(\boldsymbol{e})$. Note that the retronasal delay for $t_{\text {peak }}$ correlated significantly only with $\mathrm{P}(r=-0.93, p<0.005)$. Polar surface also showed a substantial correlation $(r=0.59)$ but not significant statistically $(p=0.11)$.

partition coefficient (P), polar surface area (polar surface), vapor pressure, density, and boiling point. The response onset delay of the retronasal route versus the orthonasal route did not correlate with vapor pressure $(r=0.18, p=0.37)$, density $(r=0.24, p=0.32)$, or boiling point $(r=-0.07, p=0.45)$, but did correlate well with polar surface $(r=0.81, p<0.05)$ and partition coefficient $(r=-0.95, p<0.005)$.
Similarly, the mean retro delay for time to peak also correlated with partition coefficient $(r=-0.93, p<0.005$; Fig. $6 \mathrm{Bb}$ ). Again, polar surface showed a positive correlation but no clear significance $(r=0.59, p=0.11)$. Vapor pressure $(r=$ $0.16, p=0.38)$, boiling point $(r=-0.16, p=0.38)$, and density $(r=0.06, p=0.46)$ did not appear to explain the variation in time to peak.

These data indicate that odorant polarity plays an important role in the length of the delay of retronasal relative to orthonasal responses. Increasing polar surface by 1 unit increases the response onset time by $3.7 \mathrm{~ms}$ and time to reach peak of retronasal responses by $5.2 \mathrm{~ms}$, relative to orthonasal responses. Increasing the odorant's polarity by $1 \log$ unit (i.e., decreasing $\mathrm{P}$ ) increases the relative retronasal onset by $\sim 49 \mathrm{~ms}$ and the time to peak by $\sim 192 \mathrm{~ms}$. Polarity also correlated positively with relative (retro delay) rise time and $t_{10}$ nearly significantly ( $p=0.06$ and $p=0.08$, respectively). Given the larger variability in the dynamics of responses to orthonasal odors (Fig. 5), we suggest that these effects of polarity are largely on orthonasal responses.

\section{Ruling out effects of phase of breathing cycle and stimulus pressure on orthonasal versus retronasal responses}

We recorded breathing and intranasal pressure signals from each animal for each route throughout the experiments. We tested whether glomerular responses to orthonasal and retronasal stimulation had any unexpected relationships with these parameters. We did not expect effects of breathing, as stimulus onset was timed independently thereof. The double-tracheotomized rats were breathing through a tracheal tube, uncoupled from the nose. The breathing frequency in animals remained uniform throughout the experiment (orthonasal: $1.60 \pm 0.11 \mathrm{~Hz}$; retronasal: $1.65 \pm 0.14 \mathrm{~Hz}$; Fig. 7). We compared stimulus onset time from the preceding inspiration (orthonasal: $0.31 \pm 0.02 \mathrm{~s}$; retronasal: $0.31 \pm 0.01 \mathrm{~s}$ ), position of stimulus onset within a breath cycle (orthonasal: $0.47 \pm 0.02$; retronasal: $0.47 \pm 0.01$; range: $0-1$ ), peak response time from the preceding inspiration (orthonasal: $1.61 \pm 0.12 \mathrm{~s}$; retronasal: $1.62 \pm 0.12 \mathrm{~s})$, and number of breath cycles until peak response (orthonasal: $2.56 \pm 0.25$; retronasal: $2.66 \pm 0.31$ ). As expected, there was no significant difference between orthonasal and retronasal trials on these parameters, suggesting that the observed effects of route on glomerular responses were not influenced by the phase of breathing cycle on average.

We also did not expect differences in intranasal pressure responses to orthonasal and retronasal trials. Indeed, the difference in the intranasal pressure parameters (maximum pressure $\left(P_{\max }\right)$, area under the pressure curve during odor stimulation $\left(P_{\text {auc }}\right)$, and the time to $P_{\max }$ could not explain the effect of route on the relative magnitudes of responses (Fig. 8). The ratios of paired averaged orthonasal and retronasal responses for each stimulus for all animals ( $\mathrm{r} / \mathrm{o} \Delta F / F ; n=106)$ did not correlate with averaged pressure ratios based on the same trials: $P_{\max }$ explained $1 \%, P_{\text {auc }} 3 \%$, and time to $P_{\max } 1 \%$ (Fig. 8). On average, $P_{\max }$ and $P_{\text {auc }}$ were somewhat below unity $(0.90 \pm 0.01)$, indicating that the retronasal flow resistance was $10 \%$ higher than the orthonasal flow. No temporal differences in flow were found.

\section{Discussion}

The spatial and temporal pattern of $\mathrm{OB}$ glomerular activity evoked by odorants is believed to represent all information about those odorants. In the present study, we investigated the effect of route on 
Table 3. Relationship between retro delay and odorant properties

\begin{tabular}{|c|c|c|c|c|c|}
\hline Temporal parameter & $\mathrm{VP}(\mathrm{mmHg})$ & $P\left(\log K_{\text {ow }}\right)$ & Density & $\mathrm{BP}\left({ }^{\circ} \mathrm{C}\right)$ & Polar surface \\
\hline Onset & $\begin{aligned} \text { Delay } & =1.3 \mathrm{VP}+94 \\
r^{2} & =0.03 \\
p & =0.37\end{aligned}$ & $\begin{aligned} \text { Delay } & =-48.8 \mathrm{P}+217 \\
r^{2} & =0.91 \\
\boldsymbol{p} & =\mathbf{0 . 0 0 2}\end{aligned}$ & $\begin{aligned} \text { Delay } & =130.5 \mathrm{D}-7 \\
r^{2} & =0.06 \\
p & =0.32\end{aligned}$ & $\begin{aligned} \text { Delay } & =-0.11 \mathrm{BP}+123 \\
r^{2} & =0.01 \\
p & =0.45\end{aligned}$ & $\begin{aligned} \text { Delay } & =3.7 \mathrm{PS}+38 \\
r^{2} & =0.65 \\
\boldsymbol{p} & =\mathbf{0 . 0 3}\end{aligned}$ \\
\hline$t_{10}$ & $\begin{aligned} \text { Delay } & =-1.88 \mathrm{VP}-28 \\
r^{2} & =0.16 \\
p & =0.22\end{aligned}$ & $\begin{aligned} \text { Delay } & =22.2 \mathrm{P}-97 \\
r^{2} & =0.44 \\
p & =0.08\end{aligned}$ & $\begin{aligned} \text { Delay } & =152 \mathrm{D}-181 \\
r^{2} & =0.18 \\
p & =0.20\end{aligned}$ & $\begin{aligned} \text { Delay } & =0.52 \mathrm{BP}-120 \\
r^{2} & =0.25 \\
p & =0.15\end{aligned}$ & $\begin{aligned} \text { Delay } & =-0.60 \mathrm{PS}-36 \\
r^{2} & =0.04 \\
p & =0.35\end{aligned}$ \\
\hline$t_{50}$ & $\begin{aligned} \text { Delay } & =0.68 \mathrm{VP}-12 \\
r^{2} & =0.06 \\
p & =0.32\end{aligned}$ & $\begin{aligned} \text { Delay } & =4.67 P-16 \\
r^{2} & =0.05 \\
p & =0.33\end{aligned}$ & $\begin{aligned} \text { Delay } & =89 D-84 \\
r^{2} & =0.17 \\
p & =0.21\end{aligned}$ & $\begin{aligned} \text { Delay } & =0.05 \mathrm{BP}+13 \\
r^{2} & =0.01 \\
p & =0.44\end{aligned}$ & $\begin{aligned} \text { Delay } & =0.34 \mathrm{PS}-12 \\
r^{2} & =0.04 \\
p & =0.36\end{aligned}$ \\
\hline$t_{90}$ & $\begin{aligned} \text { Delay } & =3.69 \mathrm{VP}+23 \\
r^{2} & =0.29 \\
p & =0.14\end{aligned}$ & $\begin{aligned} \text { Delay } & =-22.6 \mathrm{P}+112 \\
r^{2} & =0.21 \\
p & =0.18\end{aligned}$ & $\begin{aligned} \text { Delay } & =-9.5 \mathrm{D}+70 \\
r^{2} & =0.01 \\
p & =0.49\end{aligned}$ & $\begin{aligned} \text { Delay } & =-0.54 \mathrm{BP}+137 \\
r^{2} & =0.13 \\
p & =0.24\end{aligned}$ & $\begin{aligned} \text { Delay } & =1.52 \mathrm{PS}+33 \\
r^{2} & =0.12 \\
p & =0.25\end{aligned}$ \\
\hline$t_{\text {peak }}$ & $\begin{aligned} \text { Delay } & =2.24 \mathrm{VP}+137 \\
r^{2} & =0.03 \\
p & =0.38\end{aligned}$ & $\begin{aligned} \text { Delay } & =-92.1 \mathrm{P}+366 \\
r^{2} & =0.86 \\
\boldsymbol{p} & =\mathbf{0 . 0 0 4}\end{aligned}$ & $\begin{aligned} \text { Delay } & =62.3 \mathrm{D}+106 \\
r^{2} & =0.01 \\
p & =0.46\end{aligned}$ & $\begin{aligned} \text { Delay } & =-0.48 \mathrm{BP}+228 \\
r^{2} & =0.02 \\
p & =0.38\end{aligned}$ & $\begin{aligned} \text { Delay } & =5.24 \mathrm{PS}+62 \\
r^{2} & =0.35 \\
p & =0.11\end{aligned}$ \\
\hline Rise time & $\begin{aligned} \text { Delay } & =8.3 \mathrm{VP}+117 \\
r^{2} & =0.25 \\
p & =0.15\end{aligned}$ & $\begin{aligned} \text { Delay } & =-81.7 P+387 \\
r^{2} & =0.48 \\
p & =0.06\end{aligned}$ & $\begin{aligned} \text { Delay } & =-156.7 D+342 \\
r^{2} & =0.02 \\
p & =0.41\end{aligned}$ & $\begin{aligned} \text { Delay } & =-1.51 \mathrm{BP}+416 \\
r^{2} & =0.18 \\
p & =0.20\end{aligned}$ & $\begin{aligned} \text { Delay } & =4.7 P S+116 \\
r^{2} & =0.20 \\
p & =0.19\end{aligned}$ \\
\hline
\end{tabular}

VP, Vapor pressure; BP, boiling point; D, density, PS, polar surface. Values in bold are statistically significant.

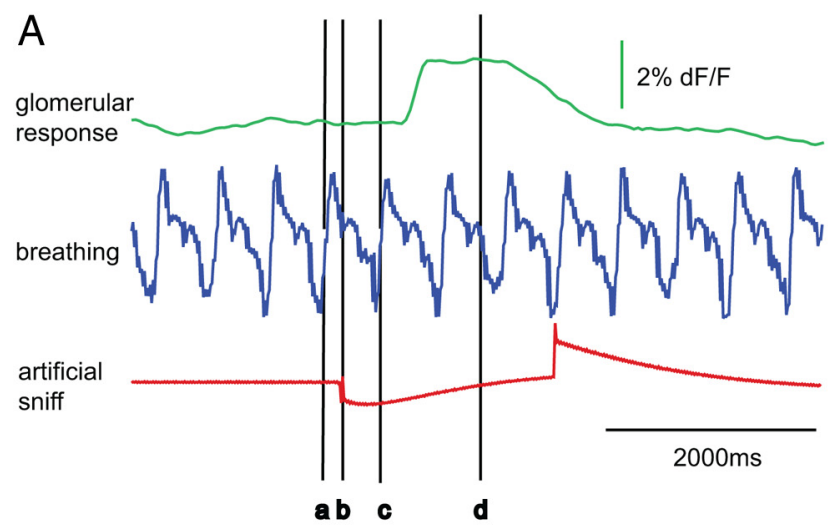

$\mathrm{a}=$ time of inspiration preceding stimulus onset $b=$ point of stimulus onset

$c=$ time of inspiration following stimulus onset

B

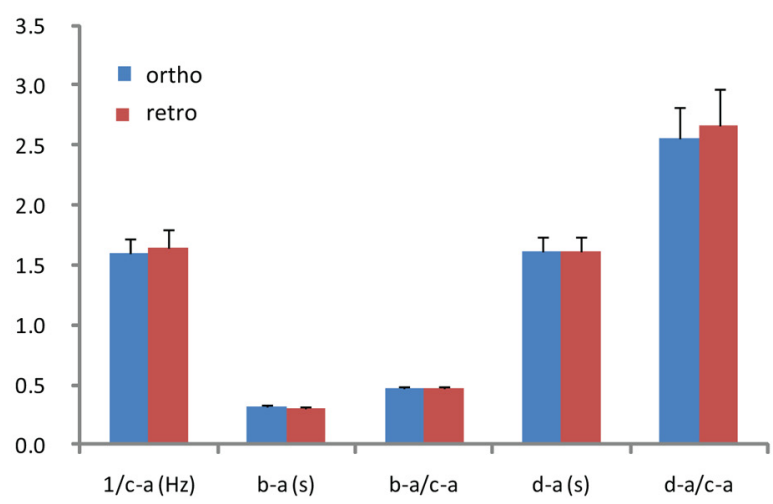

Figure 7. No coupling of orthonasal and retronasal olfactory glomerular responses with breathing. $A$, An example of the time course of a glomerular response, breathing, and artificial sniffing. Points of interest are marked with $a, b, c$, and d. $\boldsymbol{B}$, Comparison of orthonasal and retronasal breath rate $[1 /(c-a)]$, stimulus onset time from the preceding inspiration $(b-a)$, position of stimulus onset within a breath cycle $[(b-a) /(c-a)$; range: 0 to 1$]$, peak response time from the inspiration preceding stimulus onset $(d-a)$, and number of breath cycles until peak response $[(d-a) /(c-a)]$. Refer to Results for further details.

both spatial and temporal presynaptic glomerular response patterns in a rat model. To provide a mechanistic insight into the phenomena, we further examined the effects of physicochemical properties of the odorants on the orthonasal versus retronasal differences.

\section{Gross spatial activation patterns are largely independent of stimulus route}

Four aspects of the glomerular activity patterns have been proposed to contribute to an early code of odor quality in the brain: (1) the gross binary spatial glomerular response pattern, (2) the (relative) pattern of the glomerular response amplitudes, (3) the temporal dynamics, and (4) the responses relative to the phase of the respiratory cycle (Spors et al., 2006; Carey et al., 2009). To determine whether change of route would alter the glomerular response patterns, we explored each of these dimensions. First, we examined the gross (combinatorial) glomerular response patterns for each odorant stimulus and arrived at one of the basic findings of this study: retronasal stimulation did not activate a distinct set of glomeruli. Even though the magnitudes of retronasal responses were generally smaller than the orthonasal counterparts, the glomeruli activated by retronasal stimulation were not substantially different from those activated by the corresponding orthonasal stimulation (Figs. 2, 3A). Even when tiny responses $(<0.05 \% \Delta F / F)$ were considered as noise, more than two-thirds of the activated glomeruli were still common to both of the routes. Hence, we conclude that retronasal responses largely consist of the same set of activated glomeruli as orthonasal responses (Fig. $2 G$ ), and that the response differences between the routes are found elsewhere. The only caveat to this conclusion is that since we were looking only at the dorsal part of the OB, recruitment of glomeruli could occur in other areas of the $\mathrm{OB}$ in a route-dependent manner.

\section{Retronasal $\mathrm{OB}$ responses tend to be smaller than orthonasal responses}

Next, we looked into the pattern of response magnitudes across glomeruli and found that retronasal responses generally correlated positively with the orthonasal responses, but were clearly smaller in magnitude-52\% when compared pairwise (Fig. $2 G$ ) or $63 \%$ when compared ratio-wise (Fig. $3 A$ ).

If the response amplitude were the only difference between the two routes, it would raise the question of whether retronasal responses are merely equivalent to the orthonasal response to a lower concentration of the same odor. This was not the case, as further analysis revealed additional differences. For example, the retronasal versus orthonasal pattern similarity and efficacy varied across odorants and correlated only with vapor pressure, density, 
and boiling point, but not polarity/partition coefficient and polar surface (Fig. 4). This would not have been the case if retronasal responses were simply like weaker orthonasal responses.

The significant odorant-specific effects of vapor pressure, density, and boiling point on the relative magnitude of retronasal responses suggest that the more volatile the odorants are, the larger the relative retronasal responses to them tend to be. Simulations of air flow in the nasal cavity of rodents and humans suggest that retronasal flow is less effective at engaging the olfactory epithelium, especially in the caudodorsal area of the nasal cavity (Zhao et al., 2004, 2006). Thus, it is likely that a more volatile odorant may access the caudodorsal olfactory area in the nasal cavity more effectively.

Interestingly, the factors affecting odorant sorption (polarity and polar surface) did not have a significant effect on the relative retronasal response magnitudes, but did, and nearly exclusively so, have an effect on the relative temporal dynamics (see below).

\section{Retronasal temporal delay: longer response onset latency and slower rise}

Reported temporal analyses of odorant-evoked (orthonasal) input to the dorsal $\mathrm{OB}$ in rodents indicate that odorants can evoke diverse temporal patterns across activated glomeruli in an odorant-specific manner (Spors et al., 2006; Junek et al., 2010). Here, we observed that retronasal responses generally have a longer latency to onset and peak than orthonasal responses. To be sure, not only are retronasal responses slower to start, but also take more time to reach their peak level relative to this response onset (Fig. 5E). Thus, the retronasal time courses were not simply uniformly delayed (i.e., shifted rightwards; Fig. $5 E$ ), but were overall more sluggish and less robust.

These results cannot merely be the effect of weaker stimulation, because it has been shown that onset latency to orthonasal responses does not vary with odorant concentration $(0.2 \sim 5 \%$ s.v. of 2-hexanone), and there is also no consistent relationship between response latency and response amplitude (Spors et al., 2006, their Fig. 2D).

Whereas volatility (but not polarity) appeared to enhance relative retronasal efficacy, polarity (but not volatility) made the relative retronasal response more sluggish. Thus, the relative retronasal delay for the onset latency and peak response time varied across odorants and strongly correlated with odorant polarity, but not odor volatility (Fig. 6, Table 3).

The significant effects of odorant polarity and polar surface that we observed on the temporal dynamics of orthonasal versus retronasal responses across odorants are consistent with previously published literature on sorption. Odorant polarity has been recognized as a potential factor affecting dynamics of the odorant sorption in the olfactory epithelium (Mozell and Jagodowicz, 1973; Mozell et al., 1984; Palm et al., 1997; Kelder et al., 1999). Effects of odorant polar surface area on olfactory responses has not been reported yet, but polar surface properties have been reported to be predictive of intestinal absorption of drugs in humans (Palm et al., 1997; Krarup et al., 1998), and also a dominant determinant for oral absorption and brain penetration of drugs (Kelder et al., 1999).

Therefore, our data indicate that relatively slower retronasal response can be largely attributed to the odorant sorption across the olfactory epithelium. That is, the gas chromatography model of the olfactory epithelium fits nicely with the onset latencies we observed. Increasing the polarity of the odorants tended to display longer retronasal delays of 100-300 ms for the onset time and peak response time, consistent with the idea of more efficient delivery of hydrophobic compared with hydrophilic odorants in retronasal stimulation of dorsolateral ORNs in vivo (Scott et al., 2007). Our data further show that even though hydrophilic odorants reach ORNs relatively late, this delay does not affect the response magnitude.

\section{Controls: no effects of phase of breathing cycle and flow resistance}

Several reports on the orthonasal OB responses in free breathing rodents have shown coupling of glomerular responses with sniffing, and suggested that glomerular responses were modulated by breathing (Chaput et al., 1992; Spors et al., 2006). In our doubletracheotomized, artificially sniffing rats, where breathing and odor stimulation were uncoupled, orthonasal and retronasal stimulus onsets, as well as their responses, occurred on average at the same time relative to the breathing cycle (Fig. 7), implying that the observed effects of route on presynaptic glomerular responses were not due to unintended respiratory modulation.

Our setup was designed to minimize route-dependent differences between the stimuli. For example, odorant flows were generated by the same olfactometer regardless of flow direction. Stimulus onset delays were minimized by volumetrically matching the dead space of each route. We could not, however, rule out biological factors. Indeed, we did find that the retronasal flow resistance was $10 \%$ higher than the orthonasal resistance (Fig. $8 D$ ), due entirely to the flow paths inside the animal (i.e., it did not occur when we bypassed the rats). Clearly, the retronasal route has a mild flow-rectifying component. We found no evidence that this biased relative response magnitudes across the entirety of our dataset (Fig. $8 A-C$ ). 


\section{Encoding of odor route}

Data presented in this study have generated an interesting question: what biological activity of the bulb, if any, encodes the information about the odor route? It does appear that odor route can be sensed per se by humans (Small et al., 2005). We do not think route information is mediated by a subset of individual glomeruli, as we did not typically see glomeruli dedicated to a particular route (glomerulus 8 in Fig. $2 E$ is rather exceptional in that respect). In contrast, we propose that information about odor route is contained in the spatiotemporal response patterns across the entire OB. Currently, we do not know what behavior (e.g., swallow or breathing) could serve as the reference for the onset latency, and if such a reference is a prerequisite to perceptual discrimination of the direction of odor flow. It will be of interest to evaluate the perceptual effects of varying odorant volatility versus varying odorant polarity, here proposed to rather selectively affect the relative retronasal response magnitude and relative retronasal response lag, respectively. This may have important consequences for understanding the perception and neural encoding of food flavor, which is important for feeding behavior and health (Shepherd, 2012).

\section{References}

Belluscio L, Katz LC (2001) Symmetry, stereotypy, and topography of odorant representations in mouse olfactory bulbs. J Neurosci 21:2113-2122.

Bozza T, McGann JP, Mombaerts P, Wachowiak M (2004) In vivo imaging of neuronal activity by targeted expression of a genetically encoded probe in the mouse. Neuron 42:9-21.

Carey RM, Verhagen JV, Wesson DW, Pírez N, Wachowiak M (2009) Temporal structure of receptor neuron input to the olfactory bulb imaged in behaving rats. J Neurophysiol 101:1073-1088.

Chaput MA, Buonviso N, Berthommier F (1992) Temporal patterns in spontaneous and odour-evoked mitral cell discharges recorded in anaesthetized freely breathing animals. Eur J Neurosci 4:813-822.

Heilmann S, Hummel T (2004) A new method for comparing orthonasal and retronasal olfaction. Behav Neurosci 118:412-419.

Johnson BA, Leon M (2000) Modular representations of odorants in the glomerular layer of the rat olfactory bulb and the effects of stimulus concentration. J Comp Neurol 422:496-509.

Johnson BA, Leon M (2007) Chemotopic odorant coding in a mammalian olfactory system. J Comp Neur 503:1-34.

Junek S, Kludt E, Wolf F, Schild D (2010) Olfactory coding with patterns of response latencies. Neuron 67:872-884.

Kelder J, Grootenhuis PD, Bayada DM, Delbressine LP, Ploemen JP (1999) Polar molecular surface as a dominating determinant for oral absorption and brain penetration of drugs. Pharm Res 16:1514-1519.

Krarup LH, Christensen IT, Hovgaard L, Frokjaer S (1998) Predicting drug absorption from molecular surface properties based on molecular dynamics simulations. Pharm Res 15:972-978.

Lam YW, Cohen LB, Wachowiak M, Zochowski MR (2000) Odors elicit three different oscillations in the turtle olfactory bulb. J Neurosci 20:749-762.

Meister M, Bonhoeffer T (2001) Tuning and topography in an odor map on the rat olfactory bulb. J Neurosci 21:1351-1360.

Mori K, Nagao H, Yoshihara Y (1999) The olfactory bulb: coding and processing of odor molecule information. Science 286:711-715.

Mozell MM, Jagodowicz M (1973) Chromatographic separation of odor- ants by the nose: retention times measured across in vivo olfactory mucosa. Science 181:1247-1249.

Mozell MM, Sheehe PR, Swieck SW Jr, Kurtz DB, Hornung DE (1984) A parametric study of the stimulation variables affecting the magnitude of the olfactory nerve response. J Gen Physiol 83:233-267.

Palm K, Stenberg P, Luthman K, Artursson P (1997) Polar molecular surface properties predict the intestinal absorption of drugs in humans. Pharm Res 14:568-571.

Pierce J, Halpern BP (1996) Orthonasal and retronasal odorant identification based upon vapor phase input from common substances. Chem Senses 21:529-543.

Ratzlaff EH, Grinvald A (1991) A tandem-lens epifluorescence macroscope: hundred-fold brightness advantage for wide-field imaging. J Neurosci Methods 36:127-137.

Ressler KJ, Sullivan SL, Buck LB (1993) A zonal organization of odorant receptor gene expression in the olfactory epithelium. Cell 73:597-609.

Rozin P (1982) "Taste-smell confusions" and the duality of the olfactory sense. Percept Psychophys 31:397-401.

Scott JW (2006) Sniffing and temporal coding in olfaction. Chem Senses 31:119-130.

Scott JW, Acevedo HP, Sherrill L, Phan M (2007) Responses of the rat olfactory epithelium to retronasal air flow. J Neurophysiol 97:1941-1950.

Scott-Johnson PE, Blakley D, Scott JW (2000) Effects of air flow on rat electroolfactogram. Chem Senses 25:761-768.

Shepherd GM (2012) Neurogastronomy: how the brain creates flavor and why it matters, 1st edition. New York: Columbia UP.

Small DM, Gerber JC, Mak YE, Hummel T (2005) Differential neural responses evoked by orthonasal versus retronasal odorant perception in humans. Neuron 47:593-605.

Spors H, Wachowiak M, Cohen LB, Friedrich RW (2006) Temporal dynamics and latency patterns of receptor neuron input to the olfactory bulb. J Neurosci 26:1247-1259.

Sun BC, Halpern BP (2005) Identification of air phase retronasal and orthonasal odorant pairs. Chem Senses 30:693-706.

Uchida N, Takahashi YK, Tanifuji M, Mori K (2000) Odor maps in the mammalian olfactory bulb: domain organization and odorant structural features. Nat Neurosci 3:1035-1043.

Vassar R, Ngai J, Axel R (1993) Spatial segregation of odorant receptor expression in the mammalian olfactory epithelium. Cell 74:309-318.

Verhagen JV, Wesson DW, Netoff TI, White JA, Wachowiak M (2007) Sniffing controls an adaptive filter of sensory input to the olfactory bulb. Nat Neurosci 10:631-639.

Wachowiak M, Cohen LB (2001) Representation of odorants by receptor neuron input to the mouse olfactory bulb. Neuron 32:723-735.

Wachowiak M, Denk W, Friedrich RW (2004) Functional organization of sensory input to the olfactory bulb glomerulus analyzed by two-photon calcium imaging. Proc Natl Acad Sci U S A 101:9097-9102.

Wesson DW, Carey RM, Verhagen JV, Wachowiak M (2008) Rapid encoding and perception of novel odors in the rat. PLOS Biol 6:e82.

Xu F, Liu N, Kida I, Rothman DL, Hyder F, Shepherd GM (2003) Odor maps of aldehydes and esters revealed by functional MRI in the glomerular layer of the mouse olfactory bulb. Proc Natl Acad Sci U S A 100:11029-11034.

Youngentob SL, Mozell MM, Sheehe PR, Hornung DE (1987) A quantitative analysis of sniffing strategies in rats performing odor detection tasks. Physiol Behav 41:59-69.

Zhao K, Scherer PW, Hajiloo SA, Dalton P (2004) Effect of anatomy on human nasal air flow and odor transport pattern:implications for olfaction. Chem Senses 29:365-379.

Zhao K, Dalton P, Yang GC, Scherer PW (2006) Numerical modeling of turbulent and laminar airflow and odorant transport during sniffing in the human and rat nose. Chem Senses 31:107-118. 